\title{
A Sales Forecasting Model in Automotive Industry using Adaptive Neuro-Fuzzy Inference System(Anfis) and Genetic Algorithm(GA)
}

\author{
Amirmahmood Vahabi \\ Department of IT Management, \\ Science and Research branch, \\ Islamic Azad University, \\ Tehran, Iran
}

\author{
Shahrooz Seyyedi Hosseininia \\ Department of Industrial \\ Management, \\ Karaj branch, \\ Islamic Azad University, \\ Tehran, Iran
}

\author{
Mahmood Alborzi \\ Department of IT Management, \\ Science and Research branch, \\ Islamic Azad University, \\ Tehran, Iran
}

\begin{abstract}
Nowadays, Sales Forecasting is vital for any business in competitive atmosphere. For an accurate forecasting, correct variables should be considered. In this paper, we address these problems and a technique is proposed which combines two artificial intelligence algorithms in order to forecast future automobile sales in Saipa group which is a leading Automobile manufacturer in Iran. Anfis is used as the base technique which is combined with GA. GA is used in order to tune the Anfis results.

Furthermore, sales forecasting is succeeded with annual data of years between 1990 and 2016. With this in mind, per capita income, inflation rate, housing, Importation, Currency Rate (USD), loan interest rate and automobile import tariffs are selected as effective variables in the proposed model. Finally, we compare our model with ANN model which is a well-known forecasting model.
\end{abstract}

Keywords-Sales Forecasting; Adaptive Neuro-fuzzy inference system (Anfis); Genetic Algorithm (GA)

\section{INTRODUCTION}

Sale is the vital part of any business. Accordingly sales forecasting plays an important role in a business finance planning and is a self-assessment tool for a company. The managers have to keep taking the pulse of their company to know how healthy it is. A sales forecast reports, graphs and analyzes the pulse of the business. It can make the difference between just surviving and being highly successful in business. It is a vital cornerstone of a company's budget. The future direction of the company may rest on the accuracy of sales forecasting.

Nowadays, Automobile industry owns a great place in every company around the globe. As said, sales forecasting helps the company in achieving its goals such as sales revenue increasement, efficiency improvement, customer care, etc. However, still it is one of the hardest fragments of management. [1] [2]

Accurate forecasting allows the firm owners to improve market performance, gain more profit and plan its policies and procedures.

Over the last few decades when dealing with the problems of sales forecasting, traditional time series forecasting methods, such as exponential smoothing, moving average, Box Jenkins ARIMA, and multivariate regressions etc., have been proposed and widely used in practice to account for these patterns, but it always doesn't work when the market fluctuates frequently and at random [3]. Therefore, Research on novel business forecasting techniques have evoked researchers from various disciplines such as computational artificial intelligence.

Automobile market is one of the main industries in Iran which plays a vital role in country's economy. In recent years specially after sanctions which are held in 2011, Iran's automobile sales market was downsized so companies in Iran manufactured much less load than the actual production capacity. Companies' roadmap wasn't ready for this rapid change as forecasting charts wasn't able to predict these circumstances. Consequently company's revenue has dropped. In the other hand, after the joint comprehensive plan of action which was reached agreement in 2015, Iran's market experienced a new era which the market grow rapidly. Accordingly, many foreign manufacturers decided to invest in Iran market. So, in this competitive market, strategy awareness is vital. An accurate Sales forecasting model which will cover annual Sales would be a must have deal for any company which wants to have a share in the newly risen market.

In this study, we proposed a combined methodology for automobile sales forecasting in Iran. The following chapters in this article will be divided into 4 categories. Literature review and also proposed tools will be explained in chapter 2 . The proposed model and methodology, also chosen variables will be specified in chapter 3. In chapter 4 results will be shown with charts and graphs. In the end we will conclude our results in chapter 5.

\section{LITERATURE REVIEW}

Our review of literature specifies that the automobile sales forecasting study contains two types. The first is the introduction of the common forecast models presenting the sales forecast. The second is the set of studies that uses techniques to forecast the sales of automobile industry. 


\section{A. Forecasting}

Budgeting planning has an important role in any organization. The key element for a useful budgeting process is an accurate sales forecasting. Business forecasting has been continuously been a vital organization capability for both strategic and tactical business planning. [4] With this in mind, improving the quality of forecasts is still an outstanding question which will come to mind. [5] The reason that sales forecasting is particularly important is the effect that it has on many functions of the organization. [6]

Sales forecasting helps managers to make appropriate decisions in uncertain environment. Sales forecasting could be done by linear and non-linear methods. In recent years many researchers tried to develop a model to predict sales. In order to predict sales, researchers tried to use economic indexes to improve model accuracy. In 2002, Kou proposed a model and used ANN so that not only it was able to learn if-then rules, but also it could recognize the fuzzy weights. The main variable they focused on was the impact of advertising on sales [7]. Wang in 2011 proposed a model based on monthly sales in Taiwan. He used indexes such as average earning of employees in industry and services, the oil prices and the superficial measurements of housing starts and building permits, the index of producer's inventory, average monthly overtime in industry and services as the most effective variables on sales forecasting

\section{B. Artificial Intelligence Algorithms}

Statistical methods such as ARIMA and linear regression were tools for forecasters in the past. However, in the recent years, with the development of artificial intelligence models, it seems that novel tools such as ANN outperform the results given by mentioned models in the past. [8] [9] [10] [11] [12] [13]

Still, with the nearly accurate results simulated by ANN, results need to be more accurate. Accordingly, with the development of fuzzy systems and the combination with ANN, Anfis was designed which more accurate results where predicted. [14]

Development of heuristic methods, made it simpler to get results in non-exact equations. ANN technique is used in order to train Fuzzy inference systems which concludes in Anfis technique. Moreover, to get more accurate results heuristic models such as Genetic Algorithm could be used in order to tune Anfis results. In the past studies, GA was used in order to tune Anfis results which the output, outperform other compared models. [15] [16]

According to our studies, to the day, combination of Anfis and Ga isn't used in order to forecast future automobile sales in Iran. Therefore, the aim of this paper is the application of Anfis-Ga model in automobile industry sales forecasting.

\section{METHODOLOGY}

\section{A. ANN (Artificial Neural Network)}

ANN is a model for data processing which is inspired by biological nervous systems [11]. The connection between elements largely determines the network function in the nature. The key factor in this model is the proliferation of neurons whose synergy results in problem solving. ANN may consists two or more layers. In the complicated models, output of each neuron is the input for other neuron. In general ANN structure is as shown in Fig. 1.

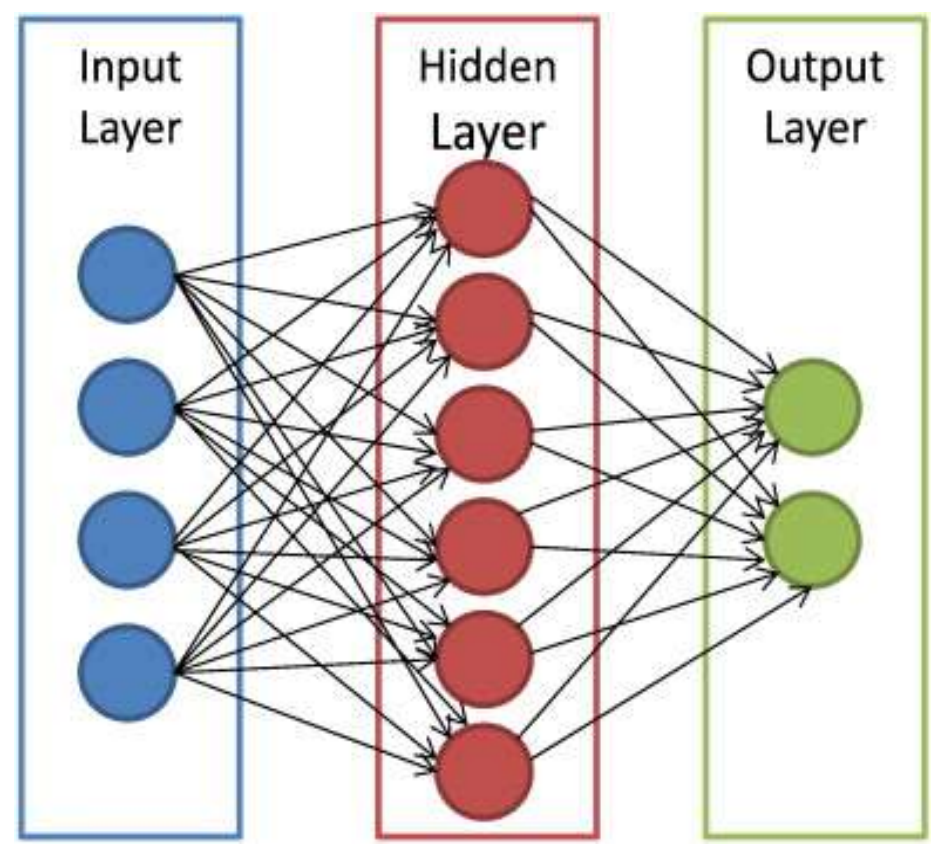

Fig. 1. ANN Structure

General of usage of ANN is for training purposes. A target function is defined and the network adjusts the output by modifying bias and weights of the network. Adjustment may occur by the comparison of output and actual targets. The network learning may continue until the outputs match targets. Normally, many individual input/target pairs are needed for network training. [14]. Usage of neural networks is also for training problems which are not conventional for computers and human knowledge.

\section{B. ANFIS (Adaptive Neuro Fuzzy Inference System)}

Anfis technique was first suggested in 1993 by Jang. [17]. Anfis is a hybrid model based in Takagi-Sugeno fuzzy inference system. One of the obstacles of the Fis technique is the extraction of fuzzy rules which when the inputs and outputs or the membership functions are occurred with multiplicity, rules extraction may face more challenging and time consuming, sometimes impossible even for expert knowledge. ANN learning technique is used in order to extract fuzzy rules for the Fuzzy inference system from the past data. The proposed technique is known as Anfis.

For simpler explanation, a fuzzy inference system with 2 inputs ( $\mathrm{x}$ and $\mathrm{y}$ ) and one output $(\mathrm{z})$ is considered. For a Sugeno model to organized a fuzzy if-then rule is needed which is expressed as

If $x$ is $A_{1}$ and $y$ is $B_{1}$ then $f_{1}=p_{1} x+q_{1} x+r_{1}$

Where $\mathrm{p}, \mathrm{r}$, and $\mathrm{q}$ are linear output parameters. The architecture of an Anfis consisted of 2 inputs and one output using 5 layers which is shown in Fig 2. 


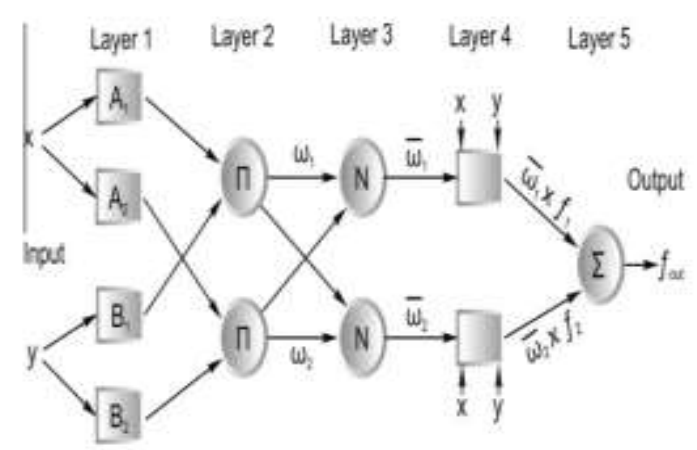

Fig. 2. Anfis structure of two inputs and two rules

Layer-1:

$O_{1, i}=\mu_{A i}(x)$, for $i=1,2,3 O_{1, i}=\mu_{B i-3}(y)$, for $i$ $=4,5,6$

Where $x$ and $y$ are node $i$ inputs, whereas $A_{i}$ and $B_{i}$ are inputs' linguistic labels. To simplify, $\mathrm{O}_{1, \mathrm{i}}$ is membership function for $\mathrm{A}_{\mathrm{i}}$ and $\mathrm{B}_{\mathrm{i}} \cdot \mu_{A i}(x)$ and $\mu_{B i}(y)$ are bell-shaped with maximum number of 1 and minimum equal to 0 , as follows:

$\mu_{A i}(x), \mu_{B i-3}(y)=\exp \left(\left(-\frac{\left(x_{i}-c_{i}\right)}{\left(a_{i}\right)}\right)^{2}\right)$

Where $\mathrm{a}_{\mathrm{i}}$ and $\mathrm{c}_{\mathrm{i}}$ is the parameter set.

Layer-2: Each node in this layer multiplies incoming inputs and send the result as output. The result represents the firing strength of the node.

$O_{2, i}=w_{i}=\mu_{A i}(x) \cdot \mu_{B i-3}(y), \quad i=1,2,3, \ldots, 9$

Layer-3: In this node the ratio of the ith rules firing strength is the sum of all rule's firing strengths is calculated.

$O_{3, i}=\bar{w}_{i}=\frac{w_{i}}{w_{1}+w_{2}+\cdots+w_{9}}, i=1,2,3, \ldots, 9$

Layer-4: Each node $I$ is a square node with a node function in this layer.

$O_{4, i}=\bar{w}_{i} . f_{i}=w_{i} .\left(p_{i} x+q_{i} x+r_{i}\right), \quad i=1,2,3, \ldots, 9$

Layer-5: In this layer, the single node is a circle node labeled $\sum$ which computes the total output as the summation of all incoming signals:

$O_{5, j}=$ overall output $=\sum_{i} \bar{w}_{i} f_{i}=\frac{\sum_{i} \bar{w}_{i} f_{i}}{\sum_{i} w_{i}}$

\section{GA (Genetic Algorithm)}

The Genetic Algorithms is categorized as a heuristic method, which is inspired by natural genetics. Mechanism is using random selections in order to improve the final result. [19] Random population is created and the use of crossover and mutation on the parent chromosomes which produces offspring that have both parents' advantages is occurred in this model. GA operation is as follows:

a) Random individuals are created which fills the initial population. All parameters could be set due to problem.

b) A fitness function must be set in order to rank the created chromosomes. Each individual is evaluated and prioritized based on the fitness measure.

c) If the termination criteria is reached, the best chromosome will be returned as the solution

d) If not, based on problem parameters, selected number of individuals from the initial population would be chosen and the genetic operations (crossover, mutation) will be applied. Newly created population would be evaluated by fitness function and all individuals would be combined and prioritized again based on fitness measures.

$e$ ) If still the termination criteria is not met, actions from step 2 will repeat until the termination criterion is satisfied. Each iteration is called generation[21-22]

In the proposed model, GA is used in order to tune the Anfis output. The elements of the Fis which is obtained from Anfis output would be extracted and would be set as chromosome model. Random individuals are created based on the model and also the number range of the genes is set by the problem parameters.

\section{RESEARCH DESIGN AND EXPERIMENTS}

This paper presents an automobile sales forecasting model based on Anfis and GA. The presented model is tested by Saipa group which is a leading automobile manufacturing company in Iran. The objective of the system is to forecast future annual sales.

The methodology for the steps of training process of the system is explained on Fig. 3. 


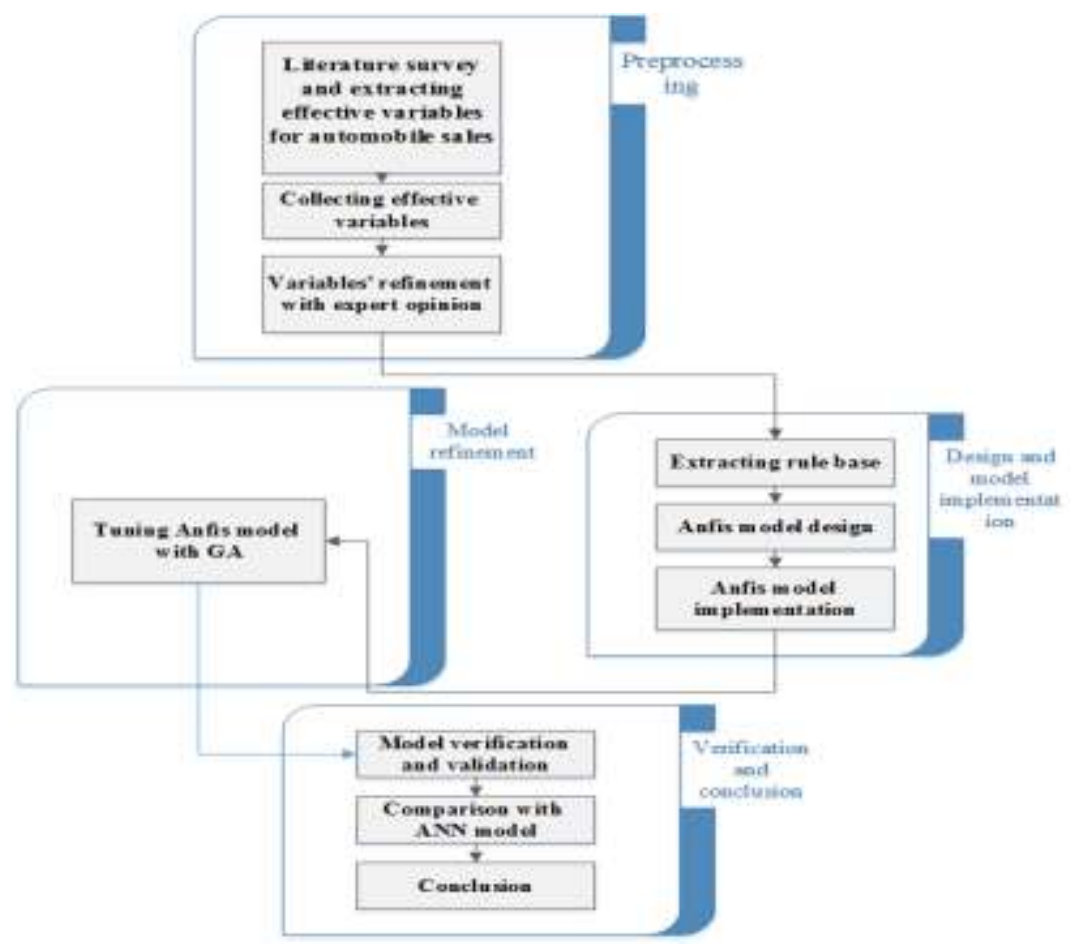

Fig. 3. Model steps

Proposed model is consisted of 4 major steps which are: 1.Preprocessing 2.Model design and implementation 3.model refinement 4 . Verification and conclusion

\section{A. Preprocessing}

From the previous studies the variables which are more effective on automobile sales are extracted. Variables such as population size, unemployment rate, exchange rates against US dollar, production index, real customs-cleared exports, the sales of manufacturing, the sales index of wholesale, the total power consumption, Consumer Price Index, Unemployment Rate, Gas Prices, Housing Starts, Gross Domestic Product, Inflations rate, Base lending rate, stock index, etc. where used in previous studies which are selected and are forwarded to next step. [18] [19] [20] [21]

Additionally, Variables per capita income, inflation rate, housing starts value, Importations value, Currency Rate (USD), loan interest rate and automobile import tariff are chosen as input for the system by experts' opinion based on checking correlations of each variable and market experience.

In this research annual data of each variable is collected from 1990 until 2005. A yearly sale of automobiles is also collected and all the data is integrated in one table and is ready for training. Sample of prepared data can be seen in Table I.

TABLE I. SAMPLE PREPARED DATA

\begin{tabular}{|c|c|c|c|c|c|c|c|c|}
\hline year & $\begin{array}{c}\text { currency } \\
\text { rate } \\
\text { against } \\
\text { USD }\end{array}$ & $\begin{array}{l}\text { inflatio } \\
n \text { rate }\end{array}$ & $\begin{array}{c}\text { per } \\
\text { capita } \\
\text { Income }\end{array}$ & $\begin{array}{c}\text { loan } \\
\text { interest } \\
\text { rate }\end{array}$ & $\begin{array}{c}\text { import } \\
\text { tariff }\end{array}$ & $\begin{array}{l}\text { importations } \\
\text { value }\end{array}$ & $\begin{array}{c}\text { housing starts } \\
\text { value }\end{array}$ & $\begin{array}{l}\text { Total } \\
\text { Sales }\end{array}$ \\
\hline 1990 & 5388.839 & $17.40 \%$ & 2228114 & 10 & 0.35 & 22013392857143 & 4766964285714 & 10894 \\
\hline 1991 & 5788.115 & $9.00 \%$ & 2795684 & 18 & 0.35 & 37053278688525 & 7992213114754 & 20878 \\
\hline 1992 & 4814.237 & $20.70 \%$ & 3183551 & 18 & 0.35 & 55372881355932 & 9982372881356 & 30859 \\
\hline 1993 & 4081.744 & $24.40 \%$ & 3094298 & 18 & 0.35 & 53324250681199 & 10391825613079 & 18536 \\
\hline 1994 & 4013.333 & $22.90 \%$ & 3869217 & 21 & 1 & 48657777777778 & 9274000000000 & 13895 \\
\hline 1995 & 4326.765 & $35.20 \%$ & 3739266 & 21 & 1 & 30568144499179 & 9141215106732 & 15642 \\
\hline
\end{tabular}




\section{B. Model design and implementation}

System is trained using Anfis technique. Input dataset is divided in two groups. $70 \%$ is chosen for training and remaining $30 \%$ is picked as test data which is used for validation. Fitness functions RMSE and $\mathrm{R}^{2}$ are used for model comparison and results evaluation.

In order to train the Anfis with most valuable data, shuffling technique used. All data is shuffled in rows before selecting train data and test data. With this method train data is chosen randomly from different years. [21]

Fuzzy C-means method is used for initial Fis generation. Default parameters are used for Anfis procedure in which initial step size is set as 0.01 . Decrease rate and increase rate for Anfis parameters are set as 0.9 and 1.1.

\section{Model refinement}

In this process, GA is used as a refinement for Anfis output results. The output Fis which is casted by Anfis training, is extracted to its core elements and labeled as $\mathrm{P}$ which gives us a $1 * \mathrm{~N}$ matrix. The extracted matrix's number of columns is modelled and a raw matrix with $\mathrm{N}$ columns is initialized which is labeled as w. Our purpose is to generate an optimum w matrix with the operations of GA which after multiplying this matrix to our base Fis elements which is $\mathrm{P}$, would give us a new matrix with $\mathrm{N}$ columns and 1 row named as $\mathrm{P}$ '. $\mathrm{P}$ ' could be transferred to a new Fis which can be evaluated by inputting data. The optimum $\mathrm{P}^{\prime}$ which is generated after termination of GA is the refined Fis which is the output of the system.

$$
P^{\prime}=P \cdot w
$$

GA parameters for tuning Anfis are set as below:

Population size: 50

Max iteration: 200

Crossover percentage: $80 \%$

Mutation percentage: $40 \%$

Selection Pressure: 8

\section{Verification and conclusion}

As stated on previous sections, $30 \%$ of data is selected as test data and is as test data isn't involved in any part of the training process, it is used as model validation. RMSE and $\mathrm{R}^{2}$ are designated as indicators for validation.

The Root Mean Square Error (RMSE) (also called the root mean square deviation, RMSD) is a commonly used measure of the difference amongst values projected by a model and the values actually observed from the modelled environment.

$$
R M S E=\sqrt{\frac{\sum_{i=1}^{n}\left(X_{o b s i}-X_{\text {model }, i}\right)^{2}}{n}}
$$

Where $X_{o b s}$ is observed values and $X_{\text {model }}$ is model evaluated values at time/place $i$.

In statistics, the coefficient of determination, denoted $R^{2}$ or $r^{2}$ and pronounced "R squared", is a number that specifies the variance in the independent variable from the dependent variable which is predictable. [23]

Based on the proportion of total variation of outcomes explained by the model, $\mathrm{R}^{2}$ provides a measure of how well observed outcomes are simulated by the model, [24]

$$
R^{2} \equiv 1-\frac{\sum_{i}\left(y_{i}-f_{i}\right)^{2}}{\sum_{i}\left(y_{i}-\bar{y}\right)^{2}}
$$

In the next step, result of the model is compared with results derived from ANN model.

\section{EMPIRICAL RESULTS}

After implementing above-mentioned data with our model, annual forecasting evaluations for automobiles based on previous yearly sales and other factors during 1990-2016 are summarized in below.

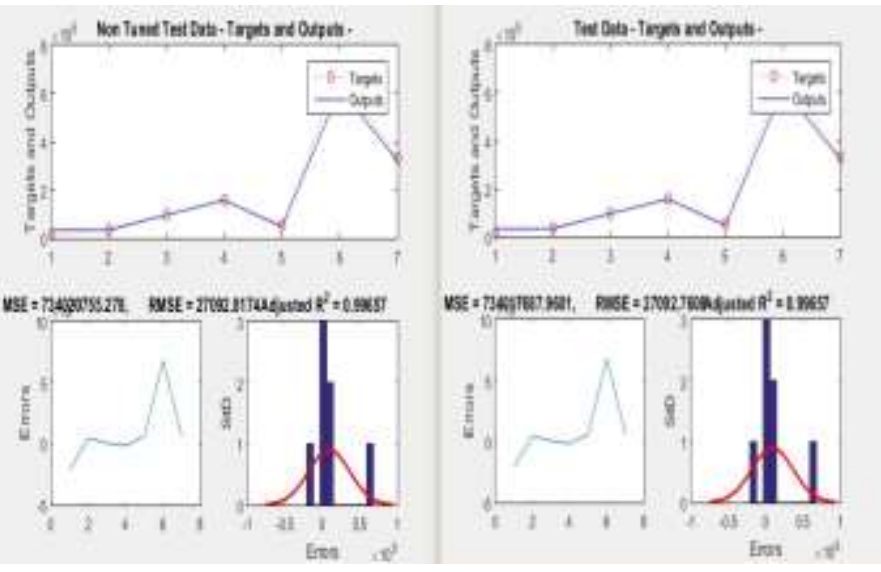

Fig. 4. Anfis-GA and Anfis model results comparison
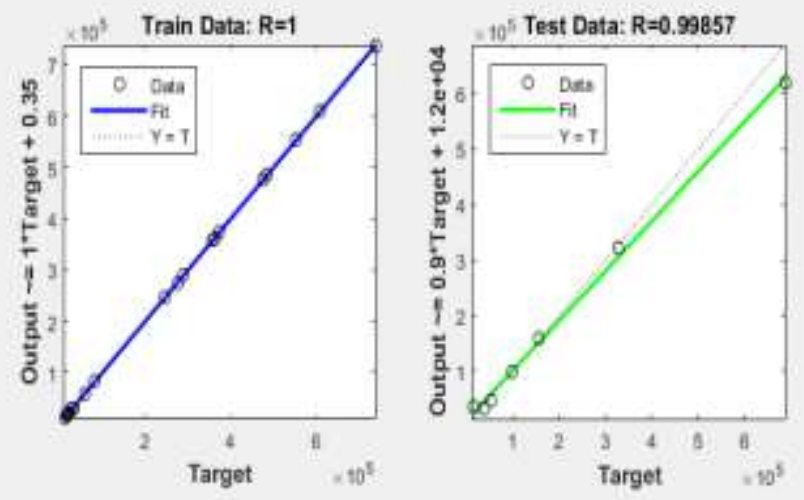

Fig. 5. Anfis results regression plot 


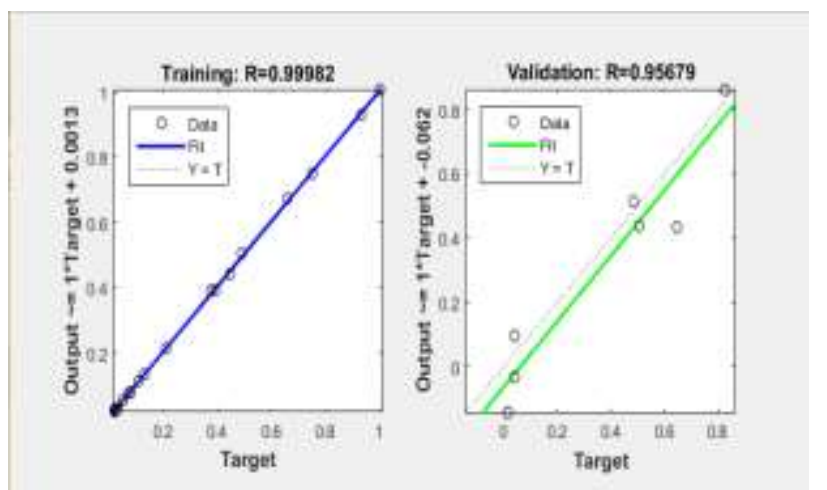

Fig. 6. ANN results regression plot

TABLE II. ANNUAL SALES DATA COMPARISON

\begin{tabular}{|l|l|l|l|l|}
\hline Year & Actual & Anfis & Anfis-GA & ANN \\
\hline 1990 & 10894 & 10894 & 10894 & 12776.3 \\
\hline 1991 & 20878 & 20878 & 20878 & 20657.1 \\
\hline 1992 & 30859 & 30858.4 & 30858.5 & 30837.6 \\
\hline 1993 & 18536 & 18536.6 & 18536.6 & 17146.9 \\
\hline 1994 & 13895 & 34936.7 & 34936.8 & 13506.6 \\
\hline 1995 & 15642 & 15642 & 15642 & 14721.4 \\
\hline 1996 & 18750 & 18750 & 18750 & 17774.1 \\
\hline 1997 & 28758 & 28759.7 & 28759.7 & 29158 \\
\hline 1998 & 38494 & 33779.1 & 33779.1 & 9567.51 \\
\hline 1999 & 53210 & 46991.6 & 46991.6 & 17879.9 \\
\hline 2000 & 57856 & 57854.3 & 57854.4 & 57367.9 \\
\hline 2001 & 81980 & 81980.5 & 81980.2 & 81496.6 \\
\hline 2002 & 97557 & 97093.3 & 97093 & 96303.4 \\
\hline 2003 & 155880 & 157490 & 157491 & 204307 \\
\hline 2004 & 245431 & 245430 & 245431 & 241285 \\
\hline 2005 & 289234 & 289235 & 289234 & 282584 \\
\hline 2006 & 372387 & 372393 & 372393 & 364094 \\
\hline 2007 & 474855 & 474849 & 474849 & 468972 \\
\hline 2008 & 484945 & 484945 & 484945 & 488537 \\
\hline 2009 & 553502 & 553504 & 553504 & 374864 \\
\hline 2010 & 608914 & 608914 & 608914 & 553111 \\
\hline 2011 & 687028 & 619231 & 619231 & 570481 \\
\hline 2012 & 736614 & 736611 & 736612 & 714066 \\
\hline 2013 & 361486 & 361484 & 361484 & 373172 \\
\hline 2014 & 275672 & 275674 & 275674 & 284691 \\
\hline 2015 & 357441 & 357441 & 357441 & 358789 \\
\hline 2016 & 328170 & 322231 & 322232 & 327672 \\
\hline & & & & \\
\hline
\end{tabular}

TABLE III. MODEL RESULTS COMPARISON

\begin{tabular}{|l|l|l|}
\hline Model & RMSE & $\mathrm{R}^{\wedge} 2$ \\
\hline Anfis & 27092.81 & $99.66 \%$ \\
\hline Anfis-GA & 27092.76 & $99.66 \%$ \\
\hline ANN & 59436.75 & $95 \%$ \\
\hline
\end{tabular}

Results obtained from Table III indicates the point that Anfis-GA model has reduced RMSE and succeeded in model tuning, although results improvement is not tangible and $\mathrm{R}^{2}$ outputs in test data hasn't changed.

\section{CONCLUSION}

This paper proposes a methodology for forecasting automobiles sales data in a manufacturing company in Iran using combination of Anfis and GA. The output system is capable of running for future forecasts. The proposed methodology has been evaluated in an automobile manufacturing company using real consumption data.
Anfis technique is used for training system as GA is used in order to tune Anfis results. Output results have been compared with ANN model and it is seen that Anfis results has outperformed ANN. With the comparison of Anfis model with Anfis-GA tuned outputs, results show that GA operates effective tuning in training procedure. Evaluated results in test data indicated the point that GA tuning may improve results. Although, improvement isn't sensed in $\mathrm{R}^{2}$ results.

Moreover, an important advantage of this methodology is that it can be updated in intervals with new data.

To conclude, in this paper our Anfis-Ga methodology is used for forecasting annual sales data in an automobile manufacturer in Iran. Results show that although using GA for tuning results outperform other results' evaluated by other models such as ANN and Anfis.

For future studies, other heuristic and meta-heuristic methods could be used for tuning Anfis results. Also Heuristic methods such as GA can be used as selection method for input variables before training with Anfis.

\section{REFERENCES}

[1] P. Chang, C. Liu and C. Fan, "Data clustering and fuzzy neural network for sales forecasting: A case study in printed circuit board industry," Knowledge-Based Systems, vol. 22, no. 5, pp. 344-355, 2009.

[2] A.-E. S. A. and F. Mannering, "Forecasting automobile demand for economics in transition, a dynamic simultaneous system approach," Transportation Planning and Technology, vol. 25, pp. 311-331, 2002.

[3] M. Lawrence and M. O’Connor, "Sales Forecasting Updates: How Good Are They in Practice?," International Journal of Forecasting, vol. 16, no. 3, pp. 369-382, 2000.

[4] R. Filders and R. Hastings, "The Organization and Improvement of Market Forecasting," Journal of Operation Research Society, vol. 45, no. 1, pp. 1-16, 1994.

[5] C. W. J. Granger and M. O'Connor, "Sales Forecasting Updates: How Good Are They in Practice?," International Journal of Forecasting, vol. 16, no. 3, pp. 369-382, 2000.

[6] J. T. Mentzer and C. C. Bienstock, Sales Forecasting Management: Understanding the Techniques, Systems and Management of the Sales Forecasting Process, Thousand Oaks, CA: SAGE Publications, Inc, 1998.

[7] R. Kou, P. Wu and C. Wang, "An intelligent sales forecasting system through integration of artificial neural networks and fuzzy neural networks with fuzzy weight elimination," Neural Networks, vol. 15, pp. 909-925, 2002.

[8] T. Kimoto, K. Asakawa, M. Yoda and M. Takeoka, "Stock market prediction system with modular neural networks," in Proceedings of the international joint conference on neural networks, San Diego, 1990.

[9] T. W. S. Chow and C. T. Leung, "Nonlinear autoregressive integrated neural network model for short-term load forecasting," IEE Proceeding Online, vol. 19960600, pp. 500-506, 1996.

[10] R. Law and N. Au, "A neural network model to forecast Japanese demand for travel to Hong Kong," Tourism Management, vol. 20, pp. 89-97, 1999.

[11] J. T. Luxh, J. O. Riis and B. Stensballe, "A hybrid econometric-neural network modeling approach for sales forecasting," The International Journal of Production Economics, vol. 43, pp. 175-192, 1996.

[12] A. S. Tawfiq and E. A. Ibrahim, "Artificial neural networks as applied to long-term demand forecasting," Artificial Intelligence in Engineering, vol. 13, pp. 189-197, 1999.

[13] F. M. Thiesing, U. Middelberg and O. Vornberger, "A neural network approach for predicting the sale of articles in supermarkets.," Third European Congress on Intelligent Techniques and Soft Computing, pp. 28-31, 1995. 
[14] A. Dwivedi, M. Niranjan and K. Sahu, "A Business Intelligence Technique for Forecasting the Automobile sales using Adaptive Intelligent Systems," International Journal of Computer Applications, vol. 74, no. 9, pp. 7-13, 2013.

[15] L.-Y. Wei, "A GA-weighted ANFIS model based on multiple stock market volatility causality for TAIEX forecasting," Applied Soft Computing, vol. 13, pp. 911-920, 2013.

[16] K. Kampouropoulos, F. Andrade, J. Cardenas and J. Romerar, "A Methodology for Enery Prediction and Optimization of a System based on the Energy Hub Concept using Particle Swarms," The Annual Seminar in Automation, Industrial Electronics and Instrumentation, 2012.

[17] J. Jang, "ANFIS: adaptive-network-based fuzzy inference system," IEEE Transactions on Systems, Man, and Cybernetics, vol. 23, no. 3, pp. 665$685,1993$.

[18] A. Sa-ngasoongsong, S. T. Bukkapatnam, J. Kim, P. S.lyer and R. Suresh, "Multi-step sales forecasting in automotive industry based on structural relationship identification," International Journal of Production Economics, vol. 140, no. 2, pp. 875-887, 2015.
[19] F.-K. Wang, K.-K. Chang and C.-W. Tzeng, "Using adaptive networkbased fuzzy inference system to forecast automobile sales," Expert Systems with Applications, vol. 38, pp. 10587-10593, 2011.

[20] F. Muhammad, M. Y. M. Hussin and A. A. Razak, "Automobile Sales and Macroeconomic Variables: A Pooled Mean Group Analysis for Asean Countries," IOSR Journal of Business and Management, pp. 1521, 2012.

[21] F.-C. Yuan, "Parameters Optimization Using Genetic Algorithms in Support Vector Regression for Sales Volume Forecasting," Applied Mathematics, vol. 3, pp. 1480-1486, 2012.

[22] M. Jalali-Heravi and A. Kyani, "Comparison of Shuffling-Adaptive Neuro Fuzzy Inference System (Shuffling-ANFIS) with Conventional ANFIS as Feature Selection Methods for Nonlinear Systems," vol. 26, no. 10 , p. $1046-1059,2007$.

[23] Stat Trek, "Stat Trek website," 2016. [Online]. Available: http://stattrek.com/.

[24] S. A. Glantz and B. K. Slinker, Primer of Applied Regression and Analysis of Variance., McGraw-Hill. ISBN 0-07-023407-8, 1990. 